P. Sivonen and A. Pärssinen, Analysis and optimization of packaged inductively degenerated common-source low-noise amplifiers with ESD protection, IEEE Transactions on Microwave Theory and Techniques, vol. 53, pp. 1304-1313, Apr. 2005.

(C) 2005 IEEE

Reprinted with permission.

This material is posted here with permission of the IEEE. Such permission of the IEEE does not in any way imply IEEE endorsement of any of Helsinki University of Technology's products or services. Internal or personal use of this material is permitted. However, permission to reprint/republish this material for advertising or promotional purposes or for creating new collective works for resale or redistribution must be obtained from the IEEE by writing to pubs-permissions@ieee.org.

By choosing to view this document, you agree to all provisions of the copyright laws protecting it. 


\title{
Analysis and Optimization of Packaged Inductively Degenerated Common-Source Low-Noise Amplifiers With ESD Protection
}

\author{
Pete Sivonen and Aarno Pärssinen, Member, IEEE
}

\begin{abstract}
The effects of packaging in inductively degenerated common-source low-noise amplifiers (LNAs) with electrostatic discharge (ESD) protection are studied and the performance of the packaged LNA is optimized. Equations describing the input impedance, transconductance, voltage gain, and noise figure (NF) of the packaged amplifier are derived and the effects of the LNA input matching network, package, and ESD parasitics on these amplifier quantities are highlighted. From the equations, several design guidelines for the packaged LNA are obtained and a systematic approach for the ESD-protected LNA optimization is deduced. It is also shown that, in the presence of an equivalent parallel package parasitic capacitance $C_{p}$, the NF in a well-optimized LNA is easily dominated by the losses of the input-matching network instead of the active device noise. Based on the theoretical results, a packaged inductively degenerated common-source LNA with ESD protection is designed in a $0.13-\mu \mathrm{m}$ CMOS process. The amplifier provides a forward gain $\left(S_{21}\right)$ of almost $18 \mathrm{~dB}$ at $2 \mathrm{GHz}$ with an NF of $1.6 \mathrm{~dB}$ while consuming $8.4 \mathrm{~mW}$ from a $1.2-\mathrm{V}$ supply.
\end{abstract}

Index Terms-CMOS, electrostatic discharge (ESD) parasitics, low-noise amplifier (LNA), packaging effects, RF.

\section{INTRODUCTION}

$\mathbf{I}$ N CONSUMER electronics applications, the integrated circuits (ICs) are almost always mounted in a package and electrostatic discharge (ESD) protection structures are required for reliability reasons. At RF frequencies, the package and ESD parasitics can have a significant effect on the circuit performance and they must be carefully taken into account in the circuit design. For the circuit simulations, accurate models for the parasitics are preferred, but analytical models are essential to develop guidelines for the circuit design and optimization.

In typical direct-conversion or low-IF receivers with an on-chip voltage-controlled oscillator (VCO) [1]-[3], the only RF off-chip interface is the low-noise amplifier (LNA) input. Thus, the package and ESD parasitics have an effect on the receiver performance only via the LNA input, assuming that a balanced LNA topology is used. Nonideal ground and supply pins have a significant effect only on the common-mode signals.

In this paper, the effects of packaging, parasitics of ESD protection structures, and the input impedance matching network on the performance of inductively degenerated common-source LNA shown in Fig. 1(a) are studied and the performance of

Manuscript received May 21, 2004; revised august 12, 2004.

P. Sivonen is with Nokia Mobile Phones, Helsinki FIN 00180, Finland (e-mail: pete.sivonen@nokia.com).

A. Pärssinen is with the Nokia Research Center, Helsinki FIN 00180, Finland Digital Object Identifier 10.1109/TMTT.2005.845773

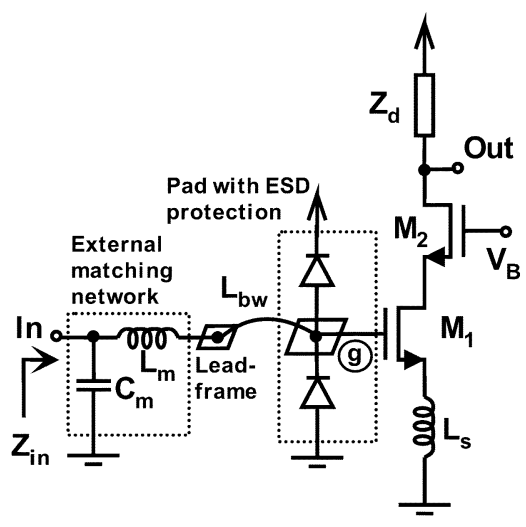

(a)

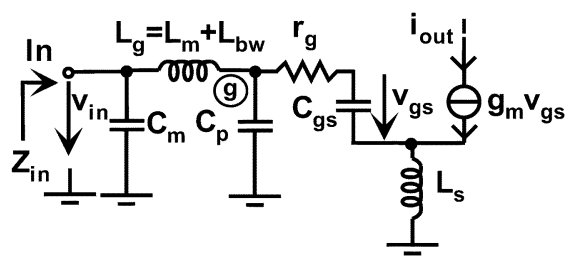

(b)

Fig. 1. (a) Single-ended equivalent circuit of packaged LNA with ESD protection. (b) Its input stage small-signal model.

the packaged LNA is optimized. Although most of the reported wireless receivers use this LNA architecture and the optimization of this topology has been extensively examined in the literature, the effects of the package and ESD parasitics are often, for simplicity, neglected in the analysis [4]-[8]. On the other hand, in most of the published studies, in which the effects of the package parasitics and ESD structures are considered, the LNA input impedance is assumed to be matched by employing only a series gate or base inductance [9]-[11]. Unfortunately, in the analysis of the package parasitics, this possesses a very restrictive constraint on the value of the equivalent parallel package parasitic capacitance $C_{p}$ [see Fig. 1(b)], which can be tolerated while still being able to match the input impedance only by series gate inductance. Namely, if $C_{p}$ is sufficiently large compared to the gate-source capacitance $C_{\mathrm{gs}}$ of the LNA input device, the impedance level at the LNA input becomes too low to be matched $50 \Omega$ only by employing a series inductance [9]-[11]. For this reason, some of the designs using only series gate inductance have selected the LNA input impedance level lower than $50 \Omega$ (i.e., 25- $\Omega$ single-ended) [11] or have simply accepted imperfect impedance match at the LNA input [12]. However, in mass-product applications, the last approach 


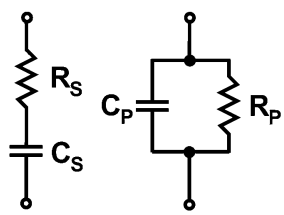

(a)

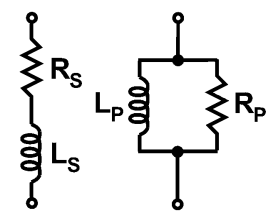

(b)
Fig. 2. Equivalent series and parallel: (a) $R C$ and (b) $R L$ circuits

is not practical since the LNA must meet its input impedancematching requirements also in the presence of process and temperature variations. If the nominal LNA $S_{11}$ is designed to be only approximately $-10 \mathrm{~dB}$, the amplifier will most probably fail to meet its matching specifications in the process corners. For this reason, the equations given in this paper assume a perfect power match at the LNA input. Moreover, in this study, an LNA input-matching network consisting of two lumped elements (see Fig. 1) is considered instead of single gate series inductance. This type of matching network allows more freedom on the value of $C_{p}$ since by employing two-component matching network, the impedance level at the LNA input can be more easily restored or matched to $50 \Omega$.

This paper is organized as follows. In Section II, the parallel-series impedance transformation technique utilized extensively in this study is revised. In Section III, the effects of the input-matching network, package, and ESD parasitics on the performance of an inductively degenerated common-source LNA are analyzed. Next, the optimization technique of the packaged LNA with ESD protection is described and an actual implementation based on the derived results is presented. Finally, the experimental results are discussed.

\section{PARALLEL-SERIES IMPEDANCE-TRANSFORMATION TECHNIQUE}

The analysis presented in this study utilizes a series of parallel-series impedance transformations at the operation frequency $f_{0}$. The parallel impedance can be replaced by a series impedance (and vice versa) if the impedances of the parallel and series versions are equal. Clearly, such a substitution cannot be valid, in general, but if the band of interest is narrow (e.g., near resonance frequency), the equivalence is reasonable [13].

The parallel-series transformation of $R C$ circuits is illustrated in Fig. 2(a). Assuming $Q^{2}=\left(\omega_{0} C_{P} R_{P}\right)^{2} \gg 1$, we have $C_{P} \approx C_{S}=C[14]$ and

$$
R_{P} \approx \frac{1}{R_{S}\left(\omega_{0} C\right)^{2}} .
$$

The parallel-series transformation of $R L$ circuits is illustrated in Fig. 2(b). In this case, the two circuits are equivalent if $Q^{2}=$ $\left(\left(\omega_{0} L_{S}\right) / R_{S}\right)^{2} \gg 1[14]$ and

$$
R_{P} \approx \frac{\left(\omega_{0} L\right)^{2}}{R_{S}}
$$

where $L_{P} \approx L_{S}=L$. It is seen that in both of the $R C$ and $R L$ cases, the transformation keeps the values of the reactive elements $C$ and $L$ nearly constant.

\section{LNA ANALYSIS}

The effects of the package parasitics, ESD protection diodes, and input-matching network on the LNA performance can be analyzed by considering the schematic shown in Fig. 1. Only the single-ended equivalent circuit of the packaged LNA with ESD protection is shown, but the results to be derived also apply to the balanced configuration. In actual implementation, a balanced LNA is used to reject the interference from the substrate and supply. The cascode transistor $M_{2}$ lowers the local oscillator (LO) leakage produced by the following mixer and improves the stability of the circuit. $M_{2}$ operates as a current-follower and is, therefore, neglected in the analysis. For the same reason, the output resistance $r_{\mathrm{ds}}$ of $M_{1}$ can be left out from the analysis.

As illustrated in Fig. 1, the bond pad, ESD diodes, Miller capacitance of $M_{1}$, and parasitic capacitance of bondwire introduce a parallel parasitic capacitance at the gate of the LNA input device $M_{1}$ (see node $g$ in Fig. 1). The total parasitic capacitance can modeled as an equivalent parallel package parasitic capacitance $C_{p}$ at the gate of $M_{1}$, as shown in Fig. 1(b), [9], [11], [12], [15], [16].

In practice, parasitic capacitance is also present in some level at the leadframe of the package. However, in this study, the leadframe capacitance $C_{\mathrm{LF}}$ is minimized by utilizing the minimum width printed circuit board (PCB) traces to route the external series inductor $L_{m}$ and the leadframe, and by placing the ground layer underneath this RF trace sufficiently deep on the multilayer PCB. Thus, in this case, $C_{\mathrm{LF}} \ll C_{p}$ and $C_{\mathrm{LF}}$ can be neglected in the analysis.

In Fig. 1, the effects of the self-inductance of the bondwire and the inductance due to the mutual inductance between the adjacent bondwires are modeled by a series inductance $L_{\mathrm{bw}}$. Since $L_{\mathrm{bw}}$ is in series with the external matching inductor $L_{m}$, the total series gate inductance is $L_{g}=L_{m}+L_{\mathrm{bw}}$. The model for the parasitics shown in Fig. 1 can be made relatively accurate provided that the adjacent pins of RF signals are grounded or otherwise properly terminated.

In Fig. $1, L_{m}$ (or $L_{g}$ ) and $C_{m}$ comprise an input impedancematching network, which is required to transform upwards the equivalent impedance looking into the gate of $M_{1}$. Since the losses of this network are crucial for the LNA noise figure (NF), these components are often realized with off-chip lumped elements.

\section{A. Input Impedance}

The effects of the input-matching network, package, and ESD parasitics on the LNA input impedance can be analyzed by considering circuit shown in Fig. 3. It should be noticed that here

$$
R_{\mathrm{eq}}=\frac{g_{m} L_{s}}{C_{\mathrm{gs}}}+r_{g} \approx \omega_{T} L_{s}
$$

represents the real part of the LNA input impedance, which is achieved in the absence of parasitics [4] (i.e., $C_{p}=0$ ). Moreover, in that particular case, the input-matching network is comprised only of a series inductance $L_{g}$ (i.e., $C_{m}=0$ ). 


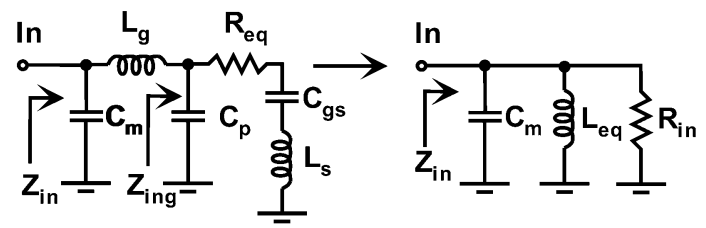

Fig. 3. LNA input impedance in the presence of parallel package parasitic capacitance $C_{p}$ is analyzed by series of parallel-series conversions.

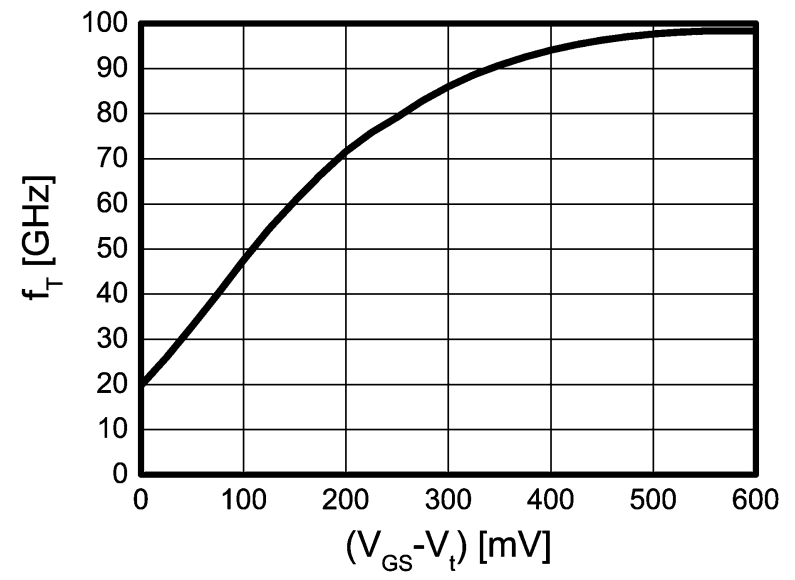

Fig. 4. Simulated unity-current gain frequency of $(\mathrm{W} / \mathrm{L})=(60 / 0.13) \mathrm{MOS}$ device.

The unity-current gain angular frequency $\omega_{T}$ of the MOS transistor can be approximated as [4]

$$
\omega_{T} \approx \frac{g_{m}}{C_{\mathrm{gs}}}=\frac{3}{2} \frac{\alpha \mu V_{\mathrm{eff}}}{L^{2}}
$$

where $\alpha=g_{m} / g_{d 0}, g_{d 0}$ is the zero bias drain conductance, $\mu$ is the electron mobility, and $V_{\text {eff }}=V_{\mathrm{GS}}-V_{T}$ is the gate-source overdrive voltage of the MOS transistor. From (4), it is concluded that the designer can control $f_{T}$ mainly via $V_{\mathrm{GS}}$ and channel length $L$.

Fig. 4 illustrates the simulated $f_{T}$ of the MOS device sized as $(\mathrm{W} / \mathrm{L})=(60 / 0.13)$ as a function of $V_{\text {eff. }}$. It is seen that for the typical overdrive voltages of $100-200 \mathrm{mV}, f_{T}$ 's in the order of $50-70 \mathrm{GHz}$ are available in the applied $0.13-\mu \mathrm{m}$ technology. In addition, since the practical values for the integrated source inductors $L_{s}$ are in the order of $1-3 \mathrm{nH}$, assuming $15-25-\mathrm{dB}$ LNA gain is to be implemented, the resulted values for $R_{\mathrm{eq}}$ are in the order of hundreds of ohms (i.e., 300-1300 $\Omega$ ). These are much larger than the traditional $50 \Omega$ considered in the older technology node $0.6 \mu \mathrm{m}$ [4]. Finally, notice that at large $V_{\text {eff }}, f_{T}$ finally saturates and ceases to increase with $V_{\text {eff. }}$.

The parallel parasitic capacitance $C_{p}$ transforms downwards the real part of the LNA input impedance. The real part of the impedance $Z_{\text {ing }}$ looking into the gate of $M_{1}$ (see Fig. 3) at the operation frequency $f_{0}$ can be approximated by

$$
\operatorname{Re}\left(Z_{\text {ing }}\right)=R_{\text {ing }}=\left(\frac{C_{\mathrm{gs}}}{C_{p}+C_{\mathrm{gs}}}\right)^{2} \cdot R_{\mathrm{eq}}
$$

where it is assumed that $\omega_{0}^{2} C_{\mathrm{gs}} L_{s} \ll 1$. This assumption can easily be verified with practical design values. Thus, due to the parasitic capacitance $C_{p}$ at the transistor gate, the input

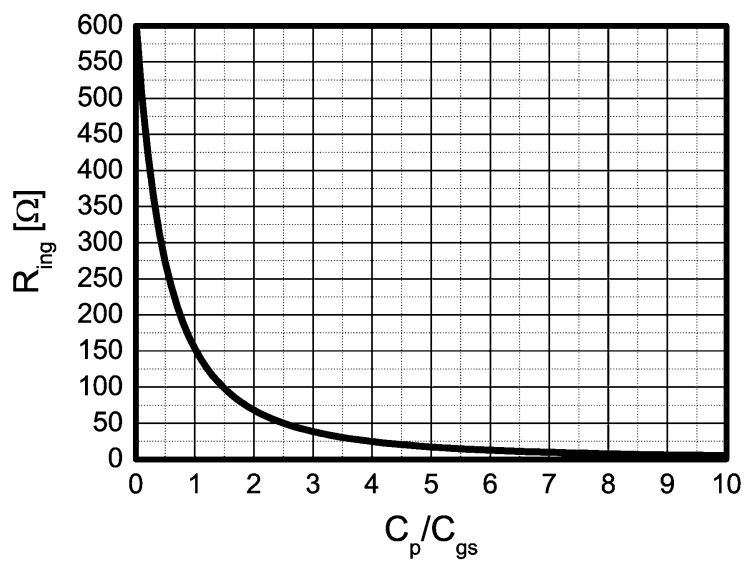

Fig. 5. Effect of package parasitic capacitance $C_{p}$ on the real part of impedance $Z_{\text {ing }}$ looking into the gate of the LNA input device $M_{1}$.

impedance comes down by a factor of $\left(C_{\mathrm{gs}} /\left(C_{p}+C_{\mathrm{gs}}\right)\right)^{2} \leq 1$ compared to the unpackaged LNA with given $\omega_{T}$ and certain size of $L_{s}$.

The effect of $C_{p}$ on $R_{\text {ing }}$ is illustrated graphically in Fig. 5. The component values used are $f_{T}=65 \mathrm{GHz}, L_{s}=1.5 \mathrm{nH}$, and $C_{\mathrm{gs}}=100 \mathrm{fF}$, giving $R_{\mathrm{eq}} \approx 610 \Omega$. The values are taken from the designed LNA. As the total parallel parasitic capacitance $C_{p}$ in this study is in the order of $500 \mathrm{fF}$, the resulted $R_{\text {ing }}$ is only approximately $20 \Omega$. Evidently, the parallel matching capacitance $C_{m}$ is needed to transform the LNA input impedance upwards to $50 \Omega$.

By applying a series of parallel-series conversions [16], it can be shown that the LNA input impedance at the operation frequency $f_{0}$ can be modeled as a parallel $C_{m} L_{\text {eq }} R_{\text {in }}$ impedance, as illustrated in Fig. 3. For the LNA input impedance to be purely real at the desired frequency of operation $f_{0}$ (i.e., $\left.Z_{\text {in }}\left(\omega_{0}\right)=R_{\text {in }}\right), C_{m}$, and $L_{\text {eq }}$ must parallel resonate at $f_{0}$

$$
\omega_{0}^{2}=\frac{1}{C_{m} L_{\mathrm{eq}}} .
$$

Then

$$
\begin{aligned}
Z_{\text {in }}\left(\omega_{0}\right) & =R_{\text {in }} \\
& =\frac{L_{\mathrm{eq}}}{C_{m}}\left(\frac{C_{p}+C_{\mathrm{gs}}}{C_{\mathrm{gs}}}\right)^{2} \cdot \frac{1}{R_{\mathrm{eq}}} \\
& =\frac{1}{\left(\omega_{0} C_{m}\right)^{2}}\left(\frac{C_{p}+C_{\mathrm{gs}}}{C_{\mathrm{gs}}}\right)^{2} \cdot \frac{1}{R_{\mathrm{eq}}}
\end{aligned}
$$

where (6) has been used. In conclusion, the value of $C_{m}$ must be selected so that $R_{\text {in }}=R_{s}$, where $R_{s}$ is the source resistance (i.e., $50 \Omega$ ). Correspondingly, the value of the series inductance $L_{g}$ must be chosen to satisfy (6) at the given $f_{0}$. Since the equivalent parallel inductance $L_{\text {eq }}$ is given as

$$
L_{\mathrm{eq}}=L_{g}-\frac{1}{\left(C_{p}+C_{\mathrm{gs}}\right) \omega_{0}^{2}}
$$

the series matching inductor $L_{g}$ must be selected as

$$
L_{g}=L_{\mathrm{eq}}+\frac{1}{\left(C_{p}+C_{\mathrm{gs}}\right) \omega_{0}^{2}}=\frac{C_{p}+C_{\mathrm{gs}}+C_{m}}{C_{m}\left(C_{p}+C_{\mathrm{gs}}\right) \omega_{0}^{2}}
$$

where (6) and (8) have been used. 


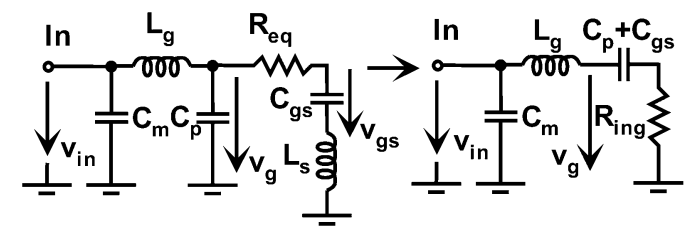

Fig. 6. Analysis of input stage transconductance $G_{m}$.

\section{B. Input Stage Transconductance}

The LNA input stage transconductance at the operation frequency $f_{0}$ can also be computed with the help of the parallel-series transformation steps, as illustrated in Fig. 6. The magnitude of the input stage output current $i_{\text {out }}$ can be approximated as

$$
\left|i_{\text {out }}\right|=g_{m}\left|v_{\mathrm{gs}}\right| \approx \frac{g_{m}\left|v_{g}\right|}{\sqrt{1+\omega_{0}^{2} C_{\mathrm{gs}}^{2} R_{\mathrm{eq}}^{2}}} \approx g_{m}\left|v_{g}\right|
$$

where it is assumed that $\omega_{0}^{2} C_{\mathrm{gs}}^{2} R_{\mathrm{eq}}^{2} \ll 1$. In addition,

$$
\left|\frac{v_{g}}{v_{\mathrm{in}}}\right|=\frac{C_{m}}{C_{p}+C_{\mathrm{gs}}} \sqrt{\frac{1+\omega_{0}^{2}\left(C_{p}+C_{\mathrm{gs}}\right)^{2} R_{\mathrm{ing}}^{2}}{1+\omega_{0}^{2} C_{m}^{2} R_{\mathrm{ing}}^{2}}} \approx \frac{C_{m}}{C_{p}+C_{\mathrm{gs}}}
$$

where (6) has been used, and it is assumed that $\omega_{0}^{2}\left(C_{p}+\right.$ $\left.C_{\mathrm{gs}}\right)^{2} R_{\text {ing }}^{2} \ll 1$ and $\omega_{0}^{2} C_{m}^{2} R_{\text {ing }}^{2} \ll 1$. Both of these assumptions can easily be verified with practical design values. Thus, the input stage transconductance at $f_{0}$ can be approximated as

$$
G_{m}=\left|\frac{i_{\mathrm{out}}}{v_{\mathrm{in}}}\right|=\frac{g_{m} C_{m}}{C_{p}+C_{\mathrm{gs}}}=\sqrt{\frac{R_{\mathrm{eq}}}{R_{s}}} \cdot \frac{1}{\omega_{0} L_{s}}
$$

where (7), (10), and (11) have been used and the LNA input impedance-matching requirement $R_{\text {in }}=R_{s}$ is applied. Since, in practice, $R_{\mathrm{eq}}$ is designed to be larger than $R_{s}$ (i.e., $50 \Omega$ ), the packaged LNA with a two-component input-matching network has a factor of $\sqrt{R_{\mathrm{eq}} / R_{s}}$ larger $G_{m}$ compared to the unpackaged LNA, in which $C_{p}$ is not present and only a series gate inductance is employed [4]. Moreover, the LNA input stage transconductance given by (12) is independent on the actual input device $g_{m}$, which is also the case in the unpackaged LNA [4]. Notice also that, in the first-order approximation, $G_{m}$ does not depend on $C_{p}$, provided that the input impedance-matching requirement is met.

Assume that the LNA drives in-phase (I) and quadrature (Q) downconversion mixers directly on-chip, as usual in direct conversion and low-IF receivers. The LNA voltage gain at $f_{0}$ is then given as

$$
A_{v}=\left|G_{m} Z_{d}\left(j \omega_{0}\right)\right| \approx \sqrt{\frac{R_{\mathrm{eq}}}{R_{s}}} \cdot \frac{\left|Z_{d}\left(j \omega_{0}\right)\right|}{\omega_{0} L_{s}}
$$

where $Z_{d}\left(j \omega_{0}\right)$ is the impedance at the drain of $M_{2}$ [see Fig. 1(a)]. Thus, the two-component input-matching network including the parallel parasitic capacitance $C_{p}$ provides an amount of $\sqrt{R_{\mathrm{eq}} / R_{s}}$ voltage gain compared to the unpackaged LNA [4].

\section{C. $N F$}

The LNA NF at the operation frequency $f_{0}$ can be estimated by analyzing the circuit shown in Fig. 7. In this circuit, $R_{\mathrm{cm}}$ and $R_{\mathrm{lg}}$ represent the series resistances of $C_{m}$ and $L_{g}$, respectively, and $R_{s}$ is the source resistance. Moreover, $\overline{i_{d}^{2}}$ is the channel

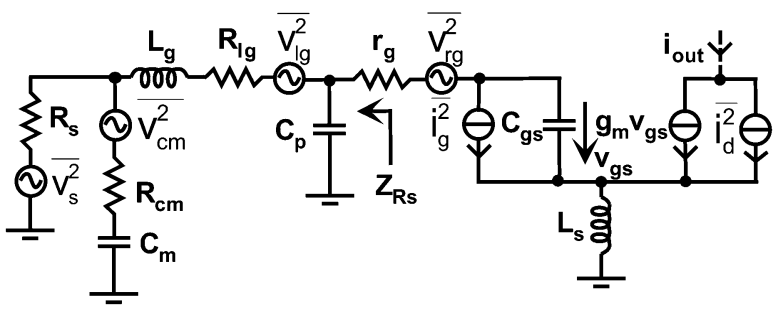

Fig. 7. Circuit model for input stage noise analysis.

thermal noise including the noise due to the substrate resistance $R_{\mathrm{sub}}$, and $\overline{i_{g}^{2}}$ is the gate-induced current noise. The analysis based on the circuit shown in Fig. 7 neglects the contributions of the cascode stage and impedance $Z_{d}$ at the drain of $M_{2}$. However, these noise contributions are considered separately.

The NF is computed by first calculating all the noise current contributions at the LNA output current. Moreover, all the calculations are done at the resonance frequency $f_{0}$ [see (6)] and assuming perfect power matching $R_{\text {in }}=R_{s}$.

From Fig. 7, the noise contributions of $R_{s}, R_{\mathrm{cm}}$, and $R_{\mathrm{lg}}$ to the LNA output noise current can be expressed as

$$
\begin{aligned}
& \overline{i_{n, R_{s}}^{2}}=\frac{G_{m}^{2} \overline{v_{n, R_{s}}^{2}}}{4}=G_{m}^{2} k_{B} T R_{s} \\
& \overline{i_{n, R_{\mathrm{cm}}}^{2}}=\frac{G_{m}^{2} \omega_{0}^{2} C_{m}^{2} R_{s}^{2} \overline{v_{n, R_{\mathrm{cm}}}^{2}}}{4}=G_{m}^{2} \omega_{0}^{2} C_{m}^{2} R_{s}^{2} k_{B} T R_{\mathrm{cm}} \\
& \overline{i_{n, R_{\mathrm{lg}}}^{2}}=\frac{G_{m}^{2} \omega_{0}^{2} C_{m}^{2} R_{s}^{2} \overline{v_{n, R_{\mathrm{lg}}}^{2}}}{4}=G_{m}^{2} \omega_{0}^{2} C_{m}^{2} R_{s}^{2} k_{B} T R_{\mathrm{lg}}
\end{aligned}
$$

respectively. Here, the input stage transconductance $G_{m}$ is defined in (12).

In order to simplify the calculation of the contributions of the rest of the noise sources, the impedance $Z_{R_{s}}$ looking into the generator (see Fig. 7) is first transformed to the equivalent series impedance by using a series of parallel-series conversions. It can be shown that, at the operation frequency $f_{0}, Z_{R_{s}}$ can be expressed as an equivalent $R L$ series impedance

$$
Z_{R_{s}}=R_{\mathrm{eq}}+j \omega_{0} \frac{L_{g} C_{m}\left(C_{p}+C_{\mathrm{gs}}\right)}{C_{\mathrm{gs}}\left(C_{p}+C_{\mathrm{gs}}+C_{p}\right)}=\omega_{T} L_{s}+\frac{j}{\omega_{0} C_{\mathrm{gs}}}
$$

where (3), (6), and (9) have been used. Consequently, by modeling $Z_{R_{s}}$ with (17) and by applying a straightforward circuit analysis, the noise contributions of $r_{g}, i_{d}$, and $i_{g}$ to the LNA output noise current can be expressed as

$$
\begin{aligned}
\overline{i_{n, r_{g}}^{2}} & =\frac{\overline{v_{n, r_{g}}^{2}}}{4\left(\omega_{0} L_{s}\right)^{2}}=\frac{k_{B} T r_{g}}{\left(\omega_{0} L_{s}\right)^{2}} \\
\overline{i_{n, d}^{2}} & =\frac{\overline{i_{d}^{2}}}{4}=k_{B} T \frac{\gamma}{\alpha} g_{m}+k_{B} T g_{\mathrm{mbs}}^{2} R_{\mathrm{sub}} \\
\overline{i_{n, i_{g}}^{2}} & =\frac{1+\left(g_{m} \omega_{0} L_{s}\right)^{2}}{4\left(\omega_{0}^{2} C_{\mathrm{gs}} L_{s}\right)^{2}} \overline{i_{g}^{2}}=\frac{1+\left(g_{m} \omega_{0} L_{s}\right)^{2}}{\left(\omega_{0} L_{s}\right)^{2}} \frac{k_{B} T \alpha \delta}{5 g_{m}}
\end{aligned}
$$

respectively. Here $g_{\mathrm{mbs}}=\eta g_{m}, \eta$ is the bias-dependent constant [17], $\gamma$ is the channel current noise factor, and $\delta$ is the gate induced current noise factor [4].

Assume that the LNA utilizes an $L C$ resonator circuit at the drain of $M_{2}$ to peek the gain of the amplifier at the frequency of 
interest $f_{0}$, as usual. If the losses of this $L C$ impedance $Z_{d}$ are modeled by a parallel resistor $R_{d}$ at $f_{0}$, the noise contribution of this resistor to the LNA output noise current is simply [6], [7]

$$
\overline{i_{n, R_{d}}^{2}}=\frac{4 k_{B} T}{R_{d}} .
$$

Finally, the LNA NF is found by adding up the squares of the different noise current contributions while taking into account the correlation between the gate and drain noise, and by normalizing the result by the noise current $\overline{i_{n, R_{s}}^{2}}$ due to $R_{s}$

$$
\begin{aligned}
\mathrm{NF}= & 1+\left(\frac{C_{p}+C_{\mathrm{gs}}}{C_{\mathrm{gs}}}\right)^{2}\left(\frac{R_{\mathrm{cm}}}{R_{\mathrm{eq}}}+\frac{R_{\mathrm{lg}}}{R_{\mathrm{eq}}}\right) \\
& +\frac{r_{g}}{R_{\mathrm{eq}}}+\frac{\gamma}{\alpha} g_{m} R_{\mathrm{eq}}\left(\frac{\omega_{0}}{\omega_{T}}\right)^{2} \Upsilon \\
& +\eta^{2} g_{m}^{2} R_{\mathrm{sub}} R_{\mathrm{eq}}\left(\frac{\omega_{0}}{\omega_{T}}\right)^{2}+\frac{\alpha \delta}{5 g_{m} R_{\mathrm{eq}}} \\
& +\frac{4 R_{\mathrm{eq}}}{R_{d}}\left(\frac{\omega_{0}}{\omega_{T}}\right)^{2} .
\end{aligned}
$$

Here, $R_{\mathrm{eq}}=\omega_{T} L_{s}$ [see (3) and (17)] represents the real part of the impedance $Z_{R_{s}}$ looking into the generator (see Fig. 7). The coefficient $\Upsilon$ is given by

$$
\Upsilon=1+2 c \sqrt{\frac{\delta}{5 \gamma}} \alpha+\frac{\delta \alpha^{2}}{5 \gamma}
$$

where $c$ is the correlation coefficient between the gate and drain noise [4].

Now the NFs of the packaged and unpackaged LNAs are compared. Recall that in the absence of the parallel package parasitic capacitance $C_{p}$, the LNA input impedance can be matched only by a series gate inductance and the amplifier NF at the operation frequency $f_{0}$ can be written as [4], [7]

$$
\begin{aligned}
\mathrm{NF}=1+ & \frac{R_{\mathrm{g}}}{R_{s}}+\frac{r_{g}}{R_{s}}+\frac{\gamma}{\alpha} g_{m} R_{s}\left(\frac{\omega_{0}}{\omega_{T}}\right)^{2} \Upsilon \\
& +\eta^{2} g_{m}^{2} R_{\mathrm{sub}} R_{s}\left(\frac{\omega_{0}}{\omega_{T}}\right)^{2}+\frac{\alpha \delta}{5 g_{m} R_{s}}+\frac{4 R_{s}}{R_{d}}\left(\frac{\omega_{0}}{\omega_{T}}\right)^{2}
\end{aligned}
$$

where $R_{s}$ is the source resistance (usually $50 \Omega$ ). It is noticed that if the losses of the LNA input-matching network are neglected and $R_{\mathrm{eq}}$ in (22) is replaced by $R_{s}$, (22) actually becomes (24).

In a general case, the comparison of the packaged and unpackaged LNA NFs is difficult. This is, for instance, due to the fact that, in the absence of $C_{p}$, the real part of the LNA input impedance is designed so that $R_{\text {in }}=\omega_{T} L_{s}=R_{s}=50 \Omega$, whereas in the presence of $C_{p}, R_{\mathrm{eq}}=\omega_{T} L_{s}>50 \Omega$, as described in Section III-A. Nevertheless, to gain insight, the NFs can be compared by assuming that, in the both unpackaged and packaged LNAs, the input devices are biased at equal $V_{\text {eff }}$. Thus, in both cases, the input transistors also have equal $\omega_{T}$. Now, in the unpackaged case, the source inductance $L_{\mathrm{su}}$ is selected so that $R_{s}=\omega_{T} L_{\mathrm{su}}=50 \Omega$ whereas in the packaged case,
$R_{\mathrm{eq}}=\omega_{T} L_{\mathrm{sp}}>50 \Omega$. Here, the subscripts $u$ and $p$ denote the unpackaged and packaged cases, respectively. Moreover, suppose that both of the LNAs draw an equal amount of current. Equivalently, in the both amplifiers, the input device widths $W$ and transconductances $g_{m}$ are equal. Finally, it is assumed that both of the amplifiers utilize equal impedances $Z_{d}$ (and, thus, $R_{d}$ ) at the drain of the cascode devices.

First neglect the losses due to the matching network components $L_{g}$ and $C_{m}$. With the assumptions described above, it is concluded that, in (22), the terms inversely proportional to $R_{\text {eq }}$ contribute less to the packaged LNA NF compared to the corresponding terms inversely proportional to $R_{s}$ in (24). On the contrary, the terms proportional to $R_{\mathrm{eq}}$ in (22) have a higher contribution to the NF compared to the corresponding terms proportional to $R_{s}$ in (24). Nevertheless, it should be noticed that all the terms in (22) describing the NF of the packaged LNA can be reduced by increasing $V_{\text {eff }}$ since both $R_{\text {eq }}$ and $\omega_{T}$ increase by increasing $V_{\text {eff }}$. Naturally, this NF reduction is done at the expense of the power consumption, assuming the LNA input device width $W$ is fixed.

From (22), it is seen that, as the parallel parasitic capacitance $C_{p}$ increases, the noise contribution due to the LNA inputmatching network increases proportional to $\left(\left(C_{\mathrm{gs}}+C_{p}\right) / C_{\mathrm{gs}}\right)^{2}$, worsening the LNA NF. On the other hand, from (24), it is noticed that, in the absence of $C_{p}$, the series resistance $R_{\lg }$ of the input-matching series inductance $L_{g}$ contributes to the LNA NF as $R_{\mathrm{lg}} / R_{s}$, where $R_{s}=50 \Omega$. Correspondingly, in the presence of $C_{p}$, the corresponding term is given as $\left(R_{\mathrm{lg}}+R_{\mathrm{cm}}\right) \cdot\left(\left(C_{\mathrm{gs}}+\right.\right.$ $\left.\left.C_{p}\right) / C_{\mathrm{gs}}\right)^{2} / R_{\mathrm{eq}}=\left(R_{\mathrm{lg}}+R_{\mathrm{cm}}\right)\left(\omega_{0} C_{m}\right)^{2} R_{s}$, which is, thus, approximately a factor of $\left(\omega_{0} C_{m}\right)^{2} R_{s}^{2}$ larger than the term in the absence of $C_{p}$ (notice that, in practice, $\left(R_{\mathrm{lg}}+R_{\mathrm{cm}}\right) \approx R_{\mathrm{lg}}$ ). For instance, in this study, the LNA input impedance matching requires that $C_{m}=3.6 \mathrm{pF}$, which, at $2 \mathrm{GHz}$, means that the noise contribution of $R_{\mathrm{lg}}$ is approximately five times larger compared to the LNA without $C_{p}$. In fact, in a well-optimized submicrometer LNA, the losses of the input-matching network can easily dominate the NF of the packaged LNA employing a two-component matching network.

Finally, as seen from (22), the NF of the packaged LNA directly depends on the parallel parasitic capacitance $C_{p}$ only via losses of the input-matching network. In principle, the value of $R_{\text {eq }}$ can be designed independently of $C_{p}$. However, in order to be able to match the LNA input impedance to $50 \Omega, R_{\mathrm{eq}}$ has to usually be designed to be larger than $50 \Omega$. In other words, the terms proportional to $R_{\text {eq }}$ together with the losses of the input-matching network usually dominate the LNA NF over the rest of the terms inversely proportional to $R_{\mathrm{eq}}$ in (22).

\section{LNA OPTIMIZATION}

In modern minimum-sized CMOS devices, the shallow trench isolation (STI) substrate resistance easily dominates over the active area substrate resistance [18] and the substrate resistance is inversely proportional to the device width $W$. On the other hand, the input device transconductance $g_{m}$ is proportional to $W$. Thus, from (22), it is seen that all 
the other terms excluding $\left(\left(C_{p}+C_{\mathrm{gs}}\right) / C_{\mathrm{gs}}\right)^{2}\left(R_{\mathrm{cm}} / R_{\mathrm{eq}}+\right.$ $\left.R_{\mathrm{lg}} / R_{\mathrm{eq}}\right),\left(4 R_{\mathrm{eq}}\right) /\left(R_{d}\right)\left(\omega_{0} / \omega_{T}\right)^{2}, \quad$ and $\quad(\alpha \delta) /\left(5 g_{m} R_{\mathrm{eq}}\right)$ increase by increasing $W$. On the contrary, since the terms $\left(\left(C_{p}+C_{\mathrm{gs}}\right) / C_{\mathrm{gs}}\right)^{2}\left(R_{\mathrm{cm}} / R_{\mathrm{eq}}+R_{\mathrm{lg}} / R_{\mathrm{eq}}\right)$ and $(\alpha \delta) /\left(5 g_{m} R_{\mathrm{eq}}\right)$ decrease by increasing $W$, an optimum input device width $W_{\text {opt }}$, which minimizes the LNA NF, exists, at least in theory. However, notice that the noise due to the gate resistance can also be minimized by interdigitating the device [4].

The optimization procedure of the packaged LNA with a twocomponent matching network is similar with the optimization of the unpackaged LNA. First, the minimum channel length provided by the given technology is selected to maximize $\omega_{T}$, given by (4), and to minimize the NF given by (22). The overdrive voltage $V_{\text {eff }}$ of the LNA input device $M_{1}$ and the value of the source inductance $L_{s}$ are then set to some arbitrary values. As $V_{\text {eff }}$ and $L_{s}$ are fixed, $R_{\text {eq }}$ is also fixed [see (3)]. In the next step, the LNA input device width $W$ is swept until an optimum NF is found. Corresponding with the each value of $W$, the input-matching network must be tuned accordingly to ensure that the LNA input impedance is matched. Finally, at this phase of the circuit design, it is reasonable to assume in the simulations that the cascode device $M_{2}$ has the same device width as the input device $M_{1}$.

As the optimum input device width $W_{\text {opt }}$ has been found, the resulted current and power consumption must be usually checked against the power consumption constraint. If the resulted power consumption is smaller than the specification, $V_{\text {eff }}$ can be increased if a smaller LNA NF is desired or required. As the increase of $V_{\text {eff }}$ increases both $\omega_{T}$ and $R_{\text {eff }}$, the NF given by (22) decreases by increasing $V_{\text {eff. }}$. On the other hand, if the resulted power consumption is higher than allowed, $V_{\text {eff }}$ must be set to a lower value and $W_{\text {opt }}$ must be re-simulated.

As $W_{\text {opt }}$ and $V_{\text {eff }}$ have been determined, the real part of the impedance $Z_{\text {ing }}$ looking into the gate of $M_{1}$ at the operation frequency can be simulated. If the resulted $R_{\text {ing }}$ is in the order of $10 \Omega$ or smaller, larger $L_{s}$ can be selected. Namely, if $R_{\text {ing }}$ is only approximately $10 \Omega$ or smaller, the input impedance matching becomes sensitive to component tolerances. If larger $L_{s}$ is needed, $W_{\text {opt }}$ must again be re-simulated.

It can be shown that the noise contribution of the LNA cascode device $M_{2}$, considering only channel thermal noise, to the LNA output noise current can be approximated as

$$
\overline{i_{n, M_{2}}^{2}}=\frac{\overline{i_{d, M_{2}}^{2}}}{1+\left(\frac{g_{m 2}}{\omega_{0} C_{\mathrm{ps}}}\right)^{2}} \approx\left(\frac{\omega_{0} C_{\mathrm{ps}}}{g_{m 2}}\right)^{2} 4 k_{B} T \frac{\gamma}{\alpha} g_{m 2}
$$

where $g_{m 2}$ is the transconductance of $M_{2}$, and $C_{\mathrm{ps}}$ is the parasitic capacitance between the drain of $M_{1}$ and the ground, respectively. Thus, the noise contribution of $M_{2}$ is minimized by minimizing the ratio $\left(\omega_{0} C_{\mathrm{ps}}\right) / g_{m 2}$ [19]. The cascode device $M_{2}$ uses the minimum channel length to minimize $C_{\mathrm{ps}}$. Moreover, since, in practice, the LNA bias current and, thus, also the drain-source current of $M_{2}$ is determined by the optimization of the LNA input device $M_{1}$, the ratio $\left(\omega_{0} C_{\mathrm{ps}}\right) / g_{m 2}$ can be minimized by selecting the width $W_{2}$ of $M_{2}$ accordingly. Often, the LNA NF is close to the minimum, when $M_{1}$ and $M_{2}$ are the same size, which gives the opportunity to merge them into one dual-gate field-effect transistor (FET) [20]. This elegant layout action further lowers the noise contribution of $M_{2}$ by lowering $C_{\mathrm{ps}}$.

As the sizes and bias points of $M_{1}$ and $M_{2}$ have been selected and the value of $L_{s}$ has been fixed, the final component values of the matching network $C_{m}$ and $L_{g}$ can be determined [see (7) and (9)]. Finally, the impedance $Z_{d}$ at the drain of $M_{2}$ is designed so that the LNA voltage gain at the operation frequency has the desired value [see (13)].

In practice, the optimization of the packaged LNA is an iterative process, in which the different design steps have an effect on each other. For instance, it might not be possible to set the LNA gain to the desired value simply by designing the impedance $Z_{d}$ accordingly since the input stage transconductance given by (12) is too small. Thus, it might be necessary to reduce the value of $L_{s}$, and this requires, for instance, that $W_{\text {opt }}$ and the component values of the matching network must be redefined. Nevertheless, the described design procedure provides guidelines for the LNA circuit design and optimization.

\section{LNA IMPLEMENTATION}

Based on theoretical results, a packaged 2-GHz inductively degenerated common-source LNA with ESD protection was designed via $0.13-\mu \mathrm{m}$ CMOS technology. In this technology, the minimum NF of a single MOS device at $2 \mathrm{GHz}$ is only in the order of $0.2 \mathrm{~dB}$. The supply voltage in this process is $1.2 \mathrm{~V}$. A balanced LNA is used to reject the interference from the substrate and supply. Although in direct conversion and low-IF receivers the LNA drives I and Q mixers directly on-chip, the LNA designed in this study was processed as a standalone circuit to be able to characterize the LNA performance separately. Thus, aside from the LNA input impedance, the LNA output impedance is also matched to a differential $100-\Omega$ impedance. The LNA is mounted in a quad flat nonleaded (QFN) package and the amplifier utilizes an RF bond pad with ESD protection diodes provided by the IC vendor. In this study, the total parasitic parallel capacitance $C_{p}$ (at nodes $g p$ and $g m$ in Fig. 8) including the capacitance due to the package parasitics, ESD protection diodes, and bond pad structure $(\sim 200 \mathrm{fF})$ is approximately $500 \mathrm{fF}$.

In the simulations, the MOS transistors were modeled by using BSIM3.v3 with parasitic gate, substrate, drain, and source resistances. The gate-induced current noise was taken into account by channel segmentation [21]. Moreover, in the simulations, the models for the PCB traces, external lumped matching components, ESD protection structures, bond pads, and package parasitics were used.

The schematic of the balanced LNA excluding the biasing details is shown in Fig. 8. At the input, the parallel matching capacitor $C_{m}$, shown in Fig. 1, is realized in a balanced LNA as a parallel component $C_{m d}=C_{m} / 2$ between the differential LNA inputs. The LNA utilizes a differential inductor $L_{d}$ of $23 \mathrm{nH}$ with a quality $(Q)$ value of $8 @ 2 \mathrm{GHz}$ to provide a high-impedance path between the supply voltage and the drain of $M_{2}$. The metal-oxide-metal (MOM) capacitors $C_{\mathrm{dc}}$ of $4 \mathrm{pF}$ ac couple the on-chip LNA output from the matching network. Finally, the output off-chip matching network consisting of the 


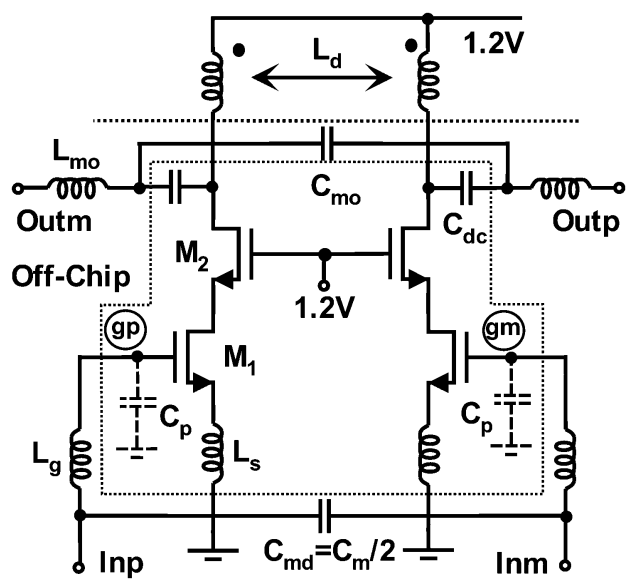

Fig. 8. Schematic of implemented LNA.

series inductor $L_{\mathrm{mo}}$ and parallel capacitance $C_{\mathrm{mo}}$ complete the LNA output matching.

As described in Section IV, the design of the LNA can be started by selecting the appropriate value for the source inductance $L_{s}$. For this purpose, the input device effective gate-source voltage $V_{\text {eff }}=\left(V_{\mathrm{GS}}-V_{t}\right)$ is set to some fixed arbitrary value, i.e., $V_{\text {eff }}=150 \mathrm{mV}$. The input device $M_{1}$ width $W_{1}$ is then swept and the LNA NF is simulated until the optimum width is found that corresponds to the minimum NF. This can be repeated for the different values of $L_{s}$. At this phase, it is reasonable to assume that the cascode width $W_{2}=W_{1}$. Moreover, corresponding the each value of $W_{1}$, the input and output matching networks are tuned to match the input and output at $2 \mathrm{GHz}$.

According to simulations, three different values of $L_{s}=$ $1.0,1.5,2.0 \mathrm{nH}$ result almost to an equal LNA noise performance at $2 \mathrm{GHz}$. On the other hand, since a low value of $L_{s}$ can imply too low a value for $R_{\text {ing }}$ [see (5)], while a high value of $L_{s}$ can result in a too low value for $G_{m}$ or LNA gain [see (12)], $L_{s}=1.5 \mathrm{nH}$ is a reasonable compromise.

As the value of $L_{s}$ is determined, an optimum input device width and bias $V_{\text {eff }}$ can be searched. Fig. 9 illustrates the simulated LNA NF at $2 \mathrm{GHz}$ with three different values of $V_{\text {eff }}=$ $50,150,250 \mathrm{mV}$ as a function of the input device width $W_{1}$. Here, $L_{s}=1.5 \mathrm{nH}, W_{2}=W_{1}$, and the LNA input and output are matched at each point.

From Fig. 9, it is seen that the increase of $V_{\text {eff }}$ from 50 to $150 \mathrm{mV}$ improves the minimum NF approximately $0.2 \mathrm{~dB}$, whereas the increase of $V_{\text {eff }}$ from 150 to $250 \mathrm{mV}$ does not result in NF improvement. Thus, it is reasonable to choose $V_{\text {eff }}=150 \mathrm{mV}$.

From Fig. 9, it is concluded that $W_{1}$ of $90-150 \mu \mathrm{m}$ results in the minimum NF corresponding to $V_{\text {eff }}=150 \mathrm{mV}$. However, since the values of $90-150 \mu \mathrm{m}$ with $V_{\text {eff }}=150 \mathrm{mV}$ correspond to a relatively high LNA current consumption of 9.7-14.3 mA, a slightly lower value of $W_{1}=60 \mu \mathrm{m}$ was selected instead. This worsens the NF only by $0.2 \mathrm{~dB}$ and corresponds to the current consumption of $7 \mathrm{~mA}$, which is tolerable on the power budget of this study.

Fig. 10 illustrates the simulated LNA NF at $2 \mathrm{GHz}$ as a function of the cascode transistor width $W_{2}$. Here, $W_{1}=60 \mu \mathrm{m}$,

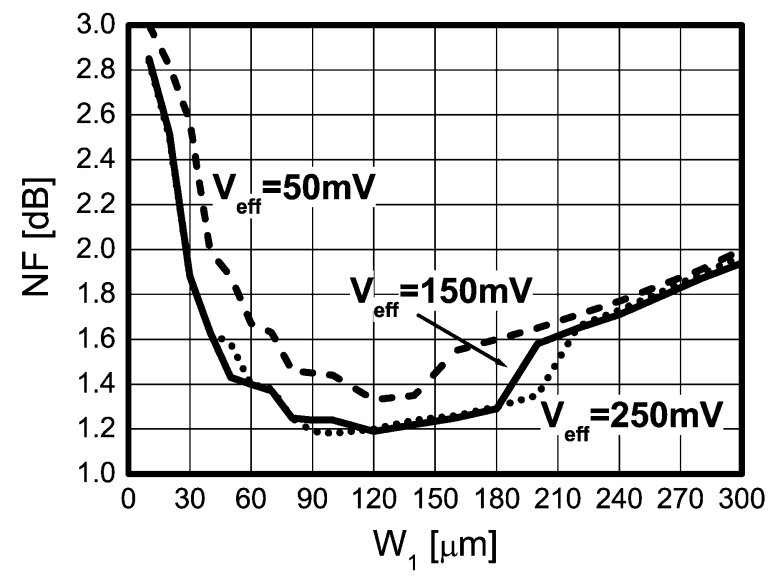

Fig. 9. Simulated LNA NF at $2 \mathrm{GHz}$ as function of input device width $W_{1}$ with three different values of $V_{\text {eff }}$.

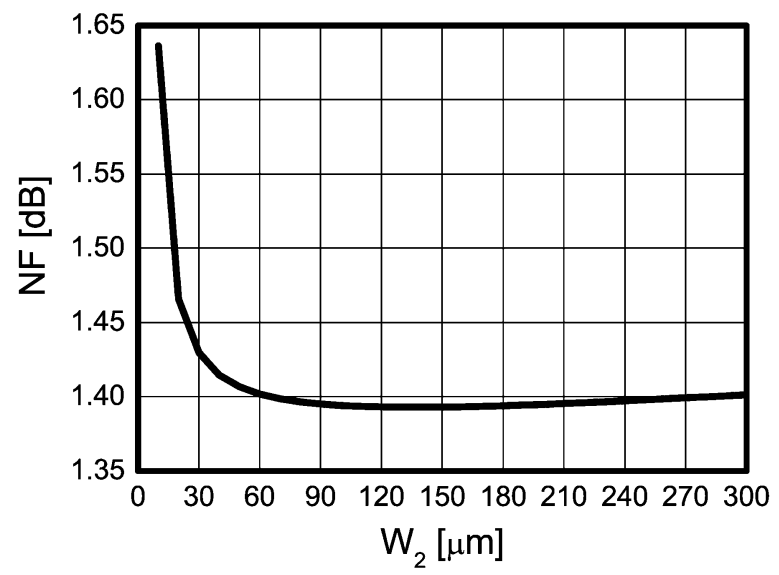

Fig. 10. Simulated LNA NF at $2 \mathrm{GHz}$ as a function of cascode transistor width $W_{2}$.

$L_{s}=1.5 \mathrm{nH}$, and $V_{\text {eff }}=150 \mathrm{mV}$. It is seen that, for the cascode widths larger than approximately $30 \mu \mathrm{m}$, the NF stays relatively constant. Notice, however, that in practice, the larger cascode width implies a larger parasitic capacitance associated with the layout wires needed to connect $M_{1}$ and $M_{2}$. Since this additional capacitance increases the noise contribution of $M_{2}$ [see (25)], it is advisable to select $W_{2}=W_{1}=60 \mu \mathrm{m}$ to simplify the layout connection of $M_{1}$ and $M_{2}$.

In order to minimize the noise contributions of the gate and substrate resistances $r_{g}$ and $R_{\text {sub }}$, respectively, it is important to select the number of gate fingers $\left(n_{f}\right)$ accordingly [4], [21]. Fig. 11 plots the LNA NF as a function of $n_{f}$. It is assumed that both $M_{1}$ and $M_{2}$ utilize an equal amount of $n_{f}$. Moreover, it is seen that $n_{f}=10$ is enough to minimize the NF. This corresponds to the gate finger width of $W_{f}=W_{1} / 10=6 \mu \mathrm{m}$. Each finger is contacted at both ends.

The final LNA utilizes the input and output matching networks with $C_{m d}=1.8 \mathrm{pF}, L_{g}=7.5 \mathrm{nH}, C_{\mathrm{mo}}=1.5 \mathrm{pF}$, and $L_{\mathrm{mo}}=9.5 \mathrm{nH}$. At $2 \mathrm{GHz}$, the amplifier has simulated $S_{11}, S_{22}<-15 \mathrm{~dB}$, and $S_{21}=19.1 \mathrm{~dB}$, input third-order intercept point IIP3 $=-6.5 \mathrm{dBm}$, and NF $=1.4 \mathrm{~dB}$. It draws approximately $7 \mathrm{~mA}$ from a $1.2-\mathrm{V}$ supply. In this case, the highest contributions to the LNA NF have the source resistance $R_{s}$ (72.0\%), the series resistance $R_{\mathrm{lg}}$ of the matching inductor $L_{g}$ 


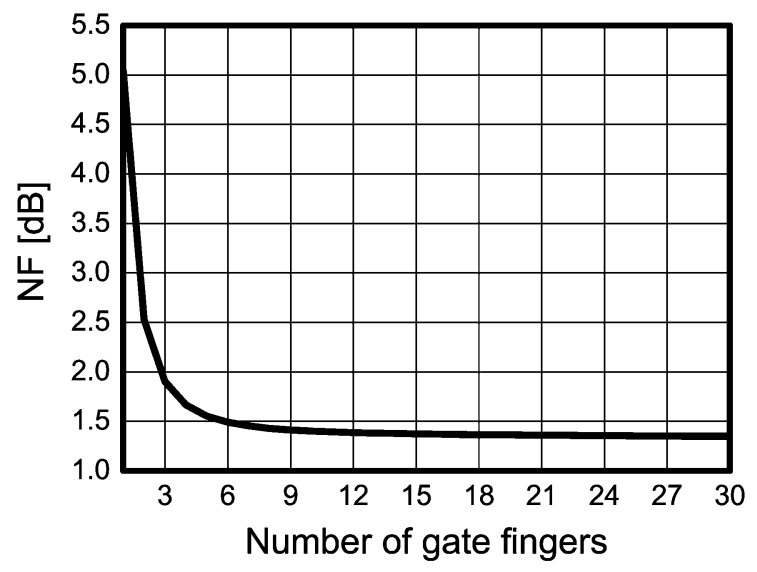

Fig. 11. Simulated LNA NF at $2 \mathrm{GHz}$ as a function of the number of gate fingers.

(13.2\%), the channel thermal noise plus gate induced current noise of $M_{1}(2.7 \%)$, the gate resistance $r_{g}$ of $M_{1}(2.0 \%)$, and the series resistance $R_{\mathrm{cm}}$ of the matching capacitor $C_{m}(1.9 \%)$. The rest of the contributions are each below $1 \%$. Notice that since the gate current noise is modeled with the channel segmentation [21], the noise contributions of the channel thermal noise and gate induced current noise cannot be separated. It is concluded that, in this study, the noise contribution of $R_{\lg }$ dominates the LNA NF, as discussed in Section IV.

Consider the effect of the equivalent parallel package parasitic capacitance $C_{p}$ on the LNA NF and $S_{21}$. Assume that the losses of the input-matching network are lumped on the equivalent series resistance $R_{\mathrm{lg}}$ of the series matching inductor $L_{g}$ $\left(R_{\mathrm{cm}}=0 \Omega\right)$. It is assumed that $R_{\mathrm{lg}}=1.3 \Omega$. For $L_{g}=7.5 \mathrm{nH}$, this corresponds to approximately a $Q$ of 72 at $2 \mathrm{GHz}$, which represents a rather typical value for $Q$ of external lumped inductors.

Fig. 12 illustrates the NF at $2 \mathrm{GHz}$ as a function of the value of $C_{p}$ in two cases. In the first case, the noise due to $R_{\mathrm{lg}}$ is set to zero in the simulator, while in the second case, the noise due to $R_{\lg }$ is considered. Again, at each point, the input and output matching networks are tuned accordingly to match the input and output impedances at $2 \mathrm{GHz}$. It is seen that, in the absence of $C_{p}$, very low LNA NFs in the order of $0.5 \mathrm{~dB}$ are available. Unfortunately, as $C_{p}$ increases, the NF increases almost linearly in decibel scale and, finally, the noise contribution of $R_{\mathrm{lg}}$ starts to dominate the NF. It is also noticed that the value of $C_{p}$ has an insignificant effect on the other LNA noise contributions since the NF with noiseless $R_{\mathrm{lg}}$ remains almost constant despite the increase of $C_{p}$. Recall that both of these conclusions can also be drawn from (22). From (22), it is seen that as $C_{p}$ increases, the noise contribution of $R_{\mathrm{g}}$ increases as $R_{\mathrm{lg}}\left(\left(C_{\mathrm{gs}}+C_{p}\right) / C_{\mathrm{gs}}\right)^{2} / R_{\text {eq }}$, while the other contributions do not depend on $C_{p}$. Thus, the LNA NF can be reduced by reducing $C_{p}$. This can be achieved partly by selecting the LNA packet pins accordingly. Nevertheless, the lower limit of $C_{p}$ is set by the ESD requirements. The noise contribution of $R_{\mathrm{lg}}$ can also be reduced by choosing larger device width and, thus, $C_{\mathrm{gs}}$ for the input device $M_{1}$. However, if the LNA current consumption is not to be increased, $V_{\text {eff }}$ must be reduced to compensate for the increase of $W_{1}$. Unfortunately, this reduces $\omega_{T}$ and

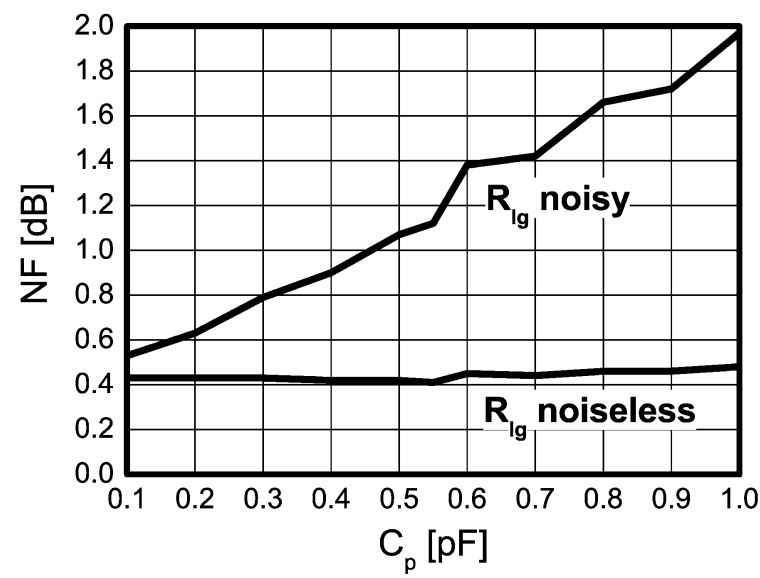

Fig. 12. Simulated LNA NF at $2 \mathrm{GHz}$ as function of equivalent parallel package parasitic capacitance $C_{p}$. Matching inductor $L_{g}$ series resistance $R_{\mathrm{lg}}=1.3 \Omega$.

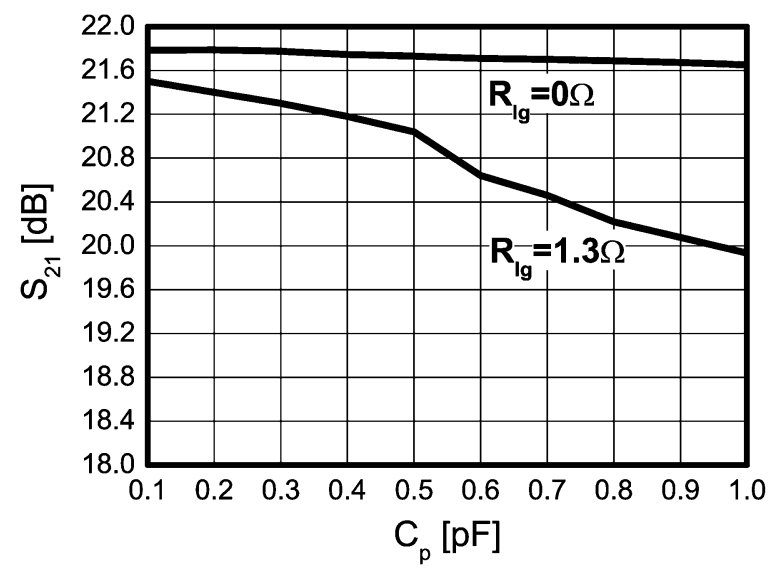

Fig. 13. Simulated LNA $S_{21}$ at $2 \mathrm{GHz}$ as a function of equivalent parallel package parasitic capacitance $C_{p}$.

$R_{\mathrm{eq}}=\omega_{T} L_{s}$ and, therefore, again, increases the noise contribution of $R_{\mathrm{lg}}$.

The effect of $C_{p}$ on the LNA available power gain $S_{21}$ at $2 \mathrm{GHz}$ is depicted in Fig. 13. Again, two cases are illustrated. First, $S_{21}$ is plotted assuming ideal series matching inductor $L_{g}$ $\left(R_{\mathrm{g}}=0 \Omega\right)$. Next, the effect of the finite inductor series resistance $\left(R_{\mathrm{lg}}=1.3 \Omega\right)$ is considered. It is seen that if $R_{\mathrm{lg}}$ is very small $\left(R_{\mathrm{lg}} \approx 0 \Omega\right), S_{21}$ stays almost constant despite the increase of $C_{p}$. Again, this agrees well with the theoretical derivations of Section III. Recall that according to (12), the LNA input stage transconductance $G_{m}$ and, thus, the voltage and power gain, do not depend on $C_{p}$ provided that $R_{\mathrm{lg}} \approx 0 \Omega$. In practice, the finite $R_{\mathrm{lg}}$ results in a slight reduction of $S_{21}$ as a function of a $C_{p}$ increment. This can also be shown by hand calculations. Nevertheless, to keep the analysis easy to interpret, $R_{\lg }$ is neglected in Section III.

\section{EXPERIMENTAL RESULTS}

The differential LNA $S$-parameters were measured by using a four-port network analyzer. The results presented here correspond to the LNA input device bias $V_{\text {eff }}=150 \mathrm{mV}$ or the LNA current consumption of $7 \mathrm{~mA}$. 


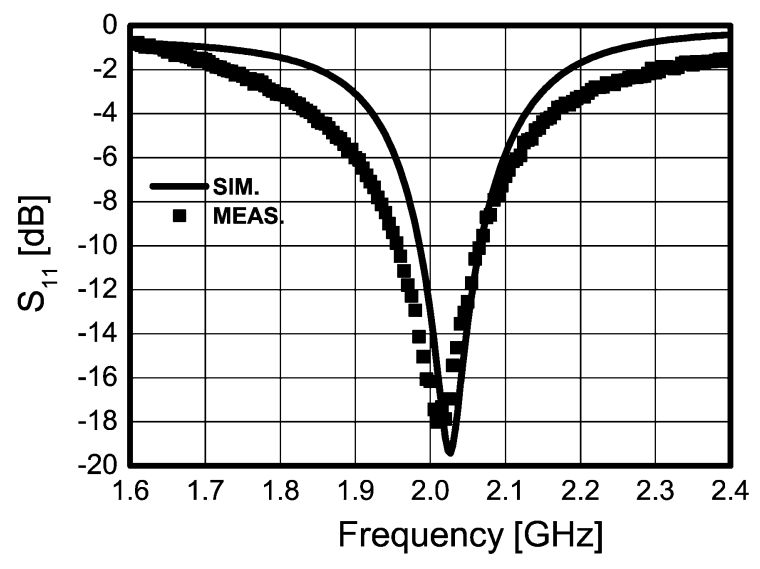

Fig. 14. Measured and simulated (solid line) LNA input impedance matching.

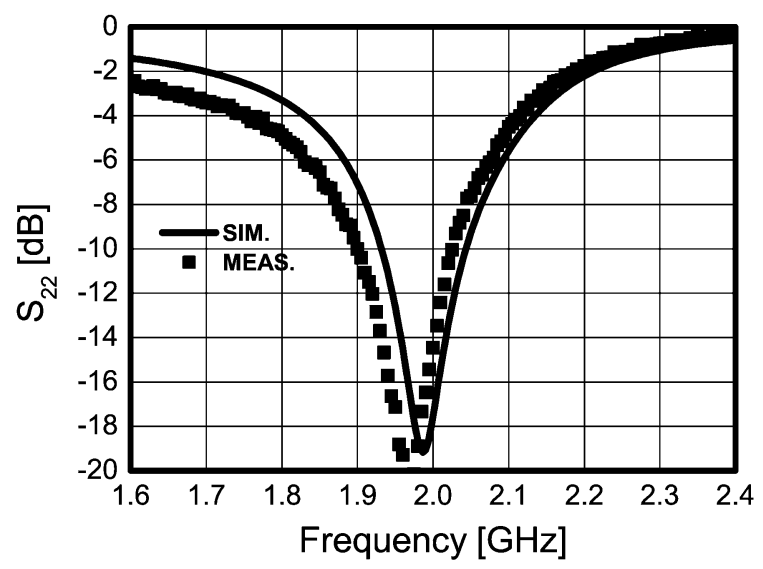

Fig. 15. Measured and simulated (solid line) LNA output impedance matching.

The measured and simulated LNA input and output scattering parameters $S_{11}$ and $S_{22}$ are shown in Figs. 14 and 15, respectively. Moreover, the measured and simulated LNA available power gains $S_{21}$ are illustrated in Fig. 16. The measured $S_{21}$ at $2 \mathrm{GHz}$ of $17.6 \mathrm{~dB}$ is approximately $1.5 \mathrm{~dB}$ smaller than expected or simulated (19.1 dB). The missing gain is due to the nonoptimized layout of the integrated differential inductor. Due to this layout failure, the $Q$ value of the inductor $L_{d}$ at the drain of $M_{2}$ is smaller than originally intended. If the simulations are rerun with the minimum $Q$ corner models, the simulated LNA $S_{21}$ is $17.8 \mathrm{~dB}$, which agrees well with the measurements. The measured IIP3 is approximately $-4.0 \mathrm{dBm}$, which is slightly higher than the simulated $-6.5 \mathrm{dBm}$. The improved linearity is due to the missing gain.

The measured and simulated LNA NFs are plotted in Fig. 17. The measured $\mathrm{NF}$ at $2 \mathrm{GHz}$ of $1.6 \mathrm{~dB}$ is approximately $0.2 \mathrm{~dB}$ higher than the nominal simulated $(1.4 \mathrm{~dB})$. It is also noticed that the simulator fails to predict the NF accurately at higher frequencies. The reason for this is uncertain.

The measured and simulated LNA NFs and forward gains as a function of the LNA current consumption are illustrated in Fig. 18. It is concluded that the NF decreases by increasing the bias current until the NF saturates at approximately $1.6 \mathrm{~dB}$. This agrees well with (22). As the increase of $V_{\text {eff }}$ or bias current at

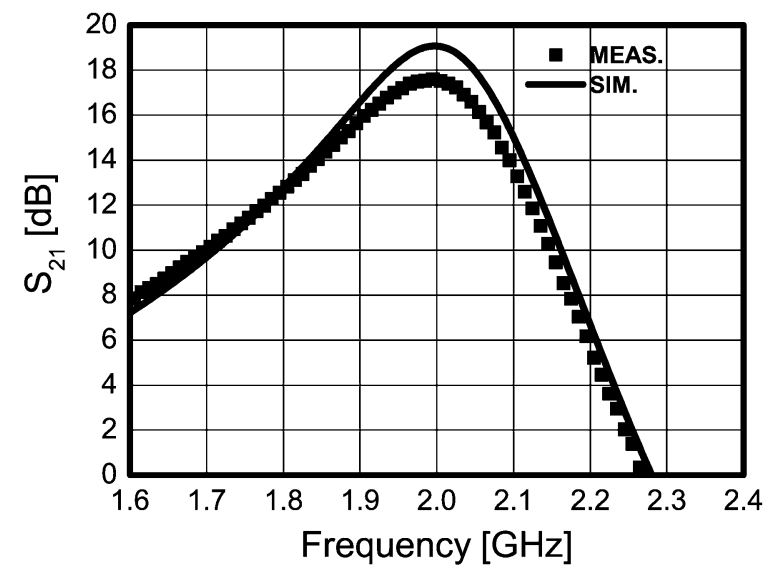

Fig. 16. Measured and simulated (solid line) LNA available power gain.

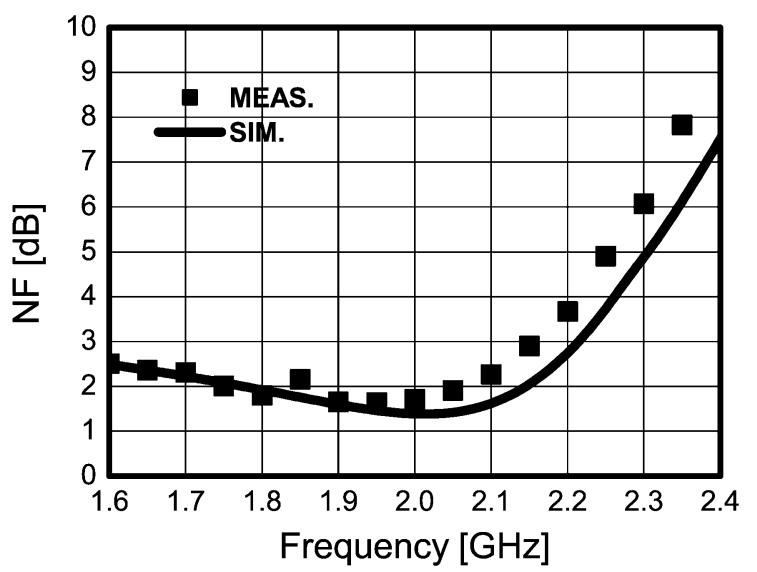

Fig. 17. Measured and simulated (solid line) LNA NF.

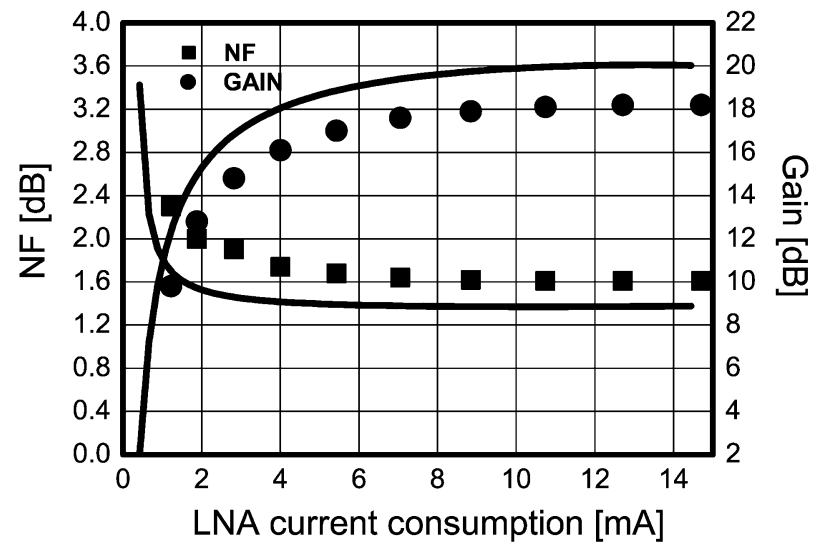

Fig. 18. Measured and simulated (solid line) LNA NF and gain.

fixed device dimensions increases both $\omega_{T}$ and $R_{\text {eff }}$, the NF decreases by increasing $V_{\text {eff. }}$. At large bias, the NF saturates due to the saturation of $\omega_{T}$ (see Fig. 4). For the same reason, both the LNA input stage $G_{m}$ and forward gain increase first by increasing $V_{\text {eff }}$ and $\omega_{T}$ [see (12)]. However, as $\omega_{T}$ saturates, $G_{m}$ and $S_{21}$ cease to increase with $V_{\text {eff. }}$. Finally, it is seen that the difference between the measured and simulated NFs increase at smaller bias currents. This is due to the fact that the used MOS 
noise model underestimates the bias-dependent gate resistance or gate induced current noise at the small gate bias.

\section{CONCLUSION}

In this paper, the effects of the package and ESD parasitics on the input matching, input stage transconductance, NF, and voltage gain of the inductively degenerated common-source LNA have been examined and the performance of the packaged LNA has been optimized. The simulations, together with the theoretical derivations, agree well with the measured LNA performance. It is concluded that, in the presence of an equivalent parallel package parasitic capacitance $C_{p}$, the NF in a well-optimized submicrometer packaged CMOS LNA is easily dominated by the losses of the input-matching network instead of the active device noise. In practical applications with ESD protection, the achievable NF of a packaged LNA is, therefore, well above the theoretical minimum NF of the active device.

\section{REFERENCES}

[1] B. Razavi, "A $900 \mathrm{MHz}$ CMOS direct conversion receiver," in VLSI Circuits Tech. Symp. Dig., 1997, pp. 113-114.

[2] A. Rofougaran, G. Chang, J. J. Rael, J. Y. C. Chang, M. Rofougaran, P. J. Chang, M. Djafari, J. Min, E. W. Roth, A. A. Abidi, and H. Samueli, "A single-chip 900-MHz spread-spectrum wireless transceiver in $1-\mu \mathrm{m}$ CMOS-Part II: Receiver design," IEEE J. Solid-State Circuits, vol. 33, no. 4, pp. 535-547, Apr. 1998.

[3] S. Tadjpour, E. Cijvat, E. Hegazi, and A. A. Abidi, "A 900-MHz dualconversion low-IF GSM receiver in 0.35- $\mu \mathrm{m}$ CMOS," IEEE J. SolidState Circuits, vol. 36, no. 12, pp. 1992-2002, Dec. 2001.

[4] D. K. Shaeffer and T. H. Lee, The Design and Implementation of LowPower CMOS Radio Receivers. Norwell, MA: Kluwer, 1999.

[5] B. A. Floyd, J. Mehta, C. Camero, and K. K. O, "A 900-MHz, $0.8 \mu \mathrm{m}$ CMOS low noise amplifier with 1.2-dB noise figure," in Proc. Custom Integrated Circuits Conf., 1999, pp. 661-664.

[6] G. Girlando and G. Palmisano, "Noise figure and impedance matching in RF cascode amplifiers," IEEE Trans. Circuits Syst. II, Analog Digit. Signal Process., vol. 46, no. 11, pp. 1388-1396, Nov. 1999.

[7] P. Andreani and H. Sjöland, "Noise optimization of an inductively degenerated CMOS low noise amplifier," IEEE Trans. Circuits Syst. II, Analog Digit. Signal Process., vol. 48, no. 9, pp. 835-841, Sep. 2001.

[8] T. K. Nguyen, C. H. Kim, G. J. Ihm, M. S. Yang, and S. G. Lee, "CMOS low-noise amplifier design optimization techniques," IEEE Trans. Microw. Theory Tech., vol. 52, no. 5, pp. 1433-1442, May 2004.

[9] P. Leroux and M. Steyaert, "High-performance $5.2 \mathrm{GHz}$ LNA with on-chip inductor to provide ESD protection," Electron. Lett., vol. 37, pp. 467-469, Mar. 2001.

[10] V. Chandrasekhar and K. Mayaram, "Analysis of CMOS RF LNA's with ESD protection," in IEEE Int. Circuits Systems Symp., 2002, pp. 779-802.

[11] P. Sivonen, S. Kangasmaa, and A. Pärssinen, "Analysis of packaging effects and optimization in inductively degenerated common-emitter lownoise amplifiers," IEEE Trans. Microw. Theory Tech., vol. 51, no. 4, pp. 1220-1226, Apr. 2003.

[12] P. Leroux, J. Janssens, and M. Steyaert, "A 0.8-dB NF ESD-protected 9-mW CMOS LNA operating at $1.23 \mathrm{GHz}$," IEEE J. Solid-State Circuits, vol. 37, no. 6, pp. 760-765, Jun. 2002.
[13] T. H. Lee, The Design of CMOS Radio-Frequency Integrated Circuits, Cambridge, U.K.: Cambridge Univ. Press, 1998, pp. 91-93.

[14] B. Razavi, RF Microelectronics. Englewood Cliffs, NJ: Prentice-Hall, 1998, pp. 50-53.

[15] G. Gramegna, M. Paparo, P. G. Erratico, and P. De Vita, "A sub-1-dB $\pm 2.3-\mathrm{kV}$ ESD-protected 900-MHz CMOS LNA," IEEE J. Solid-State Circuits, vol. 36, no. 7, pp. 1010-1017, Jul. 2001.

[16] J. Chang, "An integrated $900 \mathrm{MHz}$ spread-spectrum wireless receiver in $1-\mu \mathrm{m}$ CMOS and a suspended inductor technique," Ph.D. dissertation, Elect. Eng. Dept., UCLA, Los Angeles, CA, 1998.

[17] P. E. Allen and D. R. Holberg, CMOS Analog Circuit Design. Orlando, FL: Saunders College Publishing, 1987, pp. 114-116.

[18] R. T. Chang, M. T. Yang, P. P. C. Ho, Y. J. Wang, Y. T. Chia, B. K. Liew, C. P. Yue, and S. S. Wong, "Modeling and optimization of substrate resistance for RF-CMOS," IEEE Trans. Electron Devices, vol. 51, no. 3, pp. 421-426, Mar. 2004.

[19] W. Guo and D. Huang, "Noise and linearity analysis for a $1.9 \mathrm{GHz}$ CMOS LNA," in Proc. Asia-Pacific Circuits Systems Conf., 2002, pp. 409-414.

[20] F. Behbahani, J. C. Leete, Y. Kishigami, A. Roithmeier, K. Hoshino, and A. A. Abidi, "A 2.4-GHz low-IF receiver for wide-band WLAN in 0.6- $\mu \mathrm{m}$ CMOS-architecture and front-end," IEEE J. Solid-State Circuits, vol. 35, no. 12, Dec. 2000.

[21] A. J. Scholten, L. F. Tiemeijer, R. van Langevelde, R. J. Havens, A. T. A. Zegers-van Duijnhoven, and V. C. Venezia, "Noise modeling for RF CMOS circuit simulation," IEEE Trans. Electron Devices, vol. 50, no. 3, pp. 618-632, Mar. 2003.

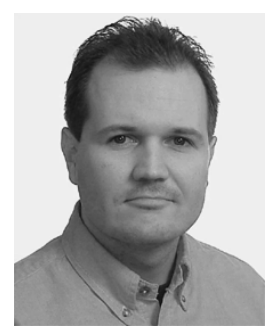

Pete Sivonen received the Master of Science (with honors) and Licentiate of Science in Technology degrees in electrical engineering from the Helsinki University of Technology (HUT), Helsinki, Finland, in 1999 and 2001, respectively.

From 1998 to 2000, he was with the Nokia Research Center, Helsinki, Finland, where he was focused on the research and design of integrated IF circuits for base-station applications. Since 2000, he has been with Nokia Mobile Phones, Helsinki, Finland, where he is currently as a Research Specialist involved with the study of integrated wireless RF transceiver front-ends. His research interests are integrated BiCMOS and CMOS analog and RF circuits, particularly for telecommunication applications.

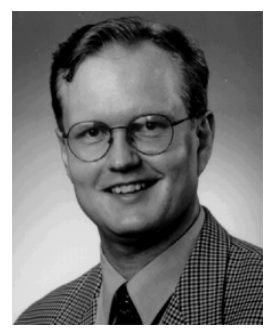

Aarno Pärssinen (S'95-M'01) received the Master of Science, Licentiate in Technology, and Doctor of Science degrees in electrical engineering from the Helsinki University of Technology, Helsinki, Finland, in 1995, 1997, and 2000, respectively.

From 1994 to 2000, he was with the Electronic Circuit Design Laboratory, Helsinki University of Technology, Finland, where he was involved with direct-conversion receivers and subsampling mixers for wireless communications. In 1996, he was a Research Visitor with the University of California at Santa Barbara. Since November 2000, he has been with the Nokia Research Center, Helsinki, Finland, where he is currently a Principal Scientist. His research interests include RF and analog IC design for wireless communications systems. 